\title{
Accelerating Progress in Regenerative Medicine by Advancing Distributed Stem Cell-based Normal Human Cell Biomanufacturing
}

\section{James L Sherley*}

The Adult Stem Cell Technology Center, LLC, Boston, Massachusetts, USA

\begin{abstract}
Successful new industries are forged in the gap between early-stage technological innovation and unmet technological needs that block new avenues to improving the quality of human life. This principle applies quite aptly to the envisioned industry of normal human cell biomanufacturing. The highly anticipated benefits of regenerative medicine have been delayed by such a technological gap. In all its varied formulations, regenerative medicine has one essential requirement, an adequate supply of normal human tissue cells for therapeutic interventions ranging from repairing debilitating injuries to treating diseases caused by tissue cell defects and deficiencies. However, with a few recent exceptions, the primary source of cells for existing and emerging regenerative therapies is direct harvest of therapeutic tissue cells from normal donors, either living or deceased. Cell supply based on donation is inherently scarce and unreliable. On-demand production of normal human tissue cells is now recognized as the ratelimiting unmet technological need that stands in the way of progress in regenerative medicine. Three different types of tissue stem cells, distributed (also known as adult), embryonic, and induced pluripotent, have been suggested as the solution for normal human tissue cell biomanufacturing. However, with currently available cell biotechnologies, only distributed stem cells (DSCs) present a realistic solution. Herein, the suitability of DSCs for advancing a new industry of normal human cell biomanufacturing is considered with respect to their pluripotent counterparts, human embryonic stem cells (ESCs) and induced pluripotent stem cells (iPSCs).
\end{abstract}

Keywords: Asymmetric self-renewal; Suppression of asymmetric cell kinetics; Purines; Drug development; Cellular therapy

\section{Introduction}

There are several early-stage innovative technologies in development that are aimed to meet the cell supply problem faced by regenerative medicine. All are based on the unique biological properties of stem cells. These technologies employ unipotent and multipotent tissue-specific distributed stem cells (DSCs; previously called "adult" stem cells), or pluripotent embryonic stem cells (ESCs) and induced pluripotent stem cells (iPSCs; Figure 1) to circumvent the intrinsic nature of normal mature functional cells - which are the ultimate units of therapeutic efficacy - not to divide, in the body or outside the body. Unlike mature cells, all three stem cell types can be propagated for long periods and cultured on-demand to high numbers (though this ability has only been established for a small number of the hundreds of tissue-specific DSC types). Thereafter, they have the potential for instruction to produce mature functional cells in comparable high numbers. However, only DSCs provide this capability free of a high risk of genetically abnormal tumor-forming cells (Figure 1). The recently introduced technology of suppression of asymmetric cell kinetics (SACK) controls the choice of DSCs either to divide symmetrically, renewing themselves, or to divide asymmetrically to produce mature functional tissue cells for tissues of important clinical need - like liver cells for drug evaluations and pancreas cells for potential treatment of type I diabetes [1-4]. Unlike ESCs and iPSCs, which form tumors, DSCs can also be re-introduced into the body for tissue restoration therapies without that worry.

Past advances in technologies for biopharmaceutical manufacturing included effective methods for large-scale production of mammalian cell lines used to produce therapeutic biomolecules and vaccines. However, in these conventional processes, the cells are a waste product that is discarded. Bridging the technological gap between early-stage innovations like SACK, for producing normal human cells on-demand, and the technological need of regenerative medicine for routine supply of normal human cells will require a new type of technological development that produces normal human cells stem and/or mature - as the desired product. Leveraging the expertise and experience of existing state of the arts human cell harvesting technologies and mammalian cell culture technologies is essential for the most effective development of a new human cell manufacturing industry that enables routine production of therapeutic human cell products. However, innovation in cell engineering, micro-fabrication and nano-fabrication, and manufacturing process engineering will also be essential for success.

\section{Regenerative Medicine Applications}

Broadly cast, regenerative medicine includes all medical modalities that require the division of human cells either in the body or outside the body. In concept and in practice, many human conditions of injury or illness can be treated or cured by replacement of the damaged or diseased human tissue cells with new normal ones. In particular, the replacement of tissue stem cells that have the ability to regenerate complete ensembles of differentiated tissue cell types can be exceptionally effective treatments. The best-known existing regenerative medicine therapy, bone marrow transplantation, has more than a half-century of clinical application. Bone marrow transplants

*Corresponding author: James L Sherley, The Adult Stem Cell Technology Center LLC, Boston, Massachusetts, USA, Tel: 617-990-6819; E-mail: jlsherley@gmail.com

Received January 15, 2014; Accepted February 19, 2014; Published February 28, 2014

Citation: Sherley JL (2014) Accelerating Progress in Regenerative Medicine by Advancing Distributed Stem Cell-based Normal Human Cell Biomanufacturing Pharm Anal Acta 5: 286. doi: 10.4172/2153-2435.1000286

Copyright: (c) 2014 Sherley JL. This is an open-access article distributed unde the terms of the Creative Commons Attribution License, which permits unrestricted use, distribution, and reproduction in any medium, provided the original author and source are credited. 


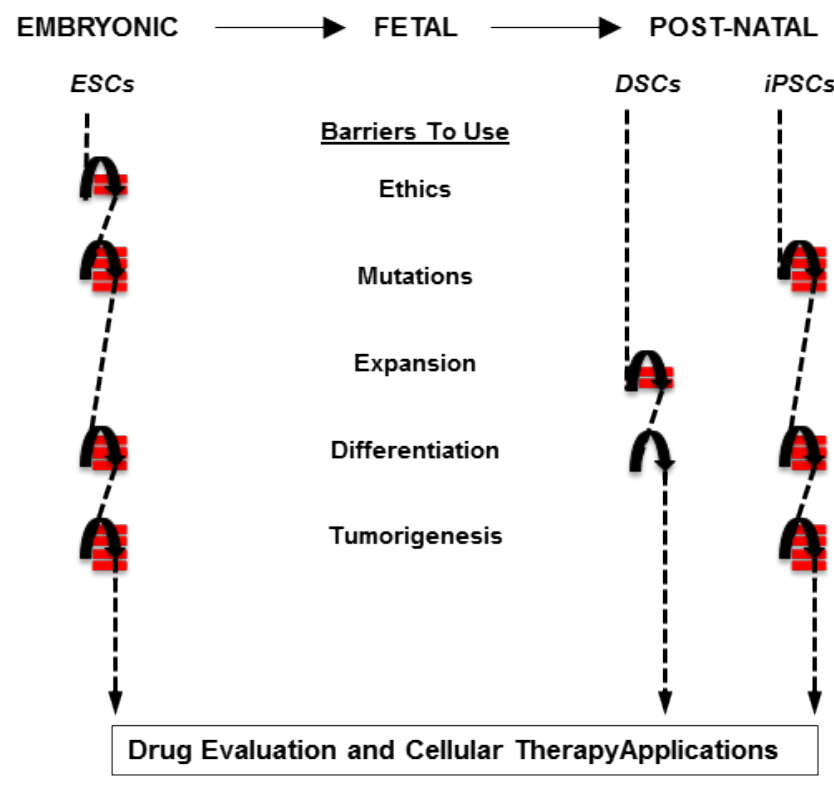

Figure 1: Barriers to the use of human stem cells for drug development and cellular therapy Human embryonic stem cells (ESCs), distributed stem cells (DSCs), and induced pluripotent stem cells (iPSCs) are derived from human tissues at the indicated stages of human development. Related to the differences in their method of derivation, the three types of human stem cells have specific profiles of barriers (Note: the height of red bricks indicates the degree of difficulty) that must be overcome (curved arrows) to achieve their successful development of applications for drug evaluation and cellular transplantation therapies.

from living donors contain hematopoietic stem cells (HSCs) that can restore complete blood cell production of a patient whose HSCs were destroyed by disease or bone marrow ablative treatments for cancer. Other well-known types of regenerative therapy are transfusions of whole blood, packed red blood cells, blood plasma, or platelets. Although these transferred human blood products do not multiply after transplantation, they were produced in the body of the donor as a result of cell division. In this manner, blood product transplants can be viewed as stem cell therapies for which the human donors are the "stem cells," who continuously produce the blood products and donate them to ill or injured recipients.

Unlike blood and blood derivatives, which are naturally rapidly replenished by the donor's body after donation, many other tissue cell types' desirable for transplantation medicine are not. Even in some cases in which they can be replenished, like liver hepatocytes, live donor transplantation can be highly risky for the donor. The lack of an adequate supply of such tissue cells motivates research to develop means for the general production of normal human tissues for transplantation medicine. In addition, these efforts are driven by the need for normal human tissues for use in pre-clinical drug evaluations. Short of phase I clinical trials to evaluate the potential toxicity of new drug candidates, human tissue cells provide the most faithful assay. In the case of liver hepatocytes, they are also essential for pre-clinical evaluations of drug metabolism and drug interactions. Since, living donors cannot be used for such cell harvests, cells from cadavers are used instead. These are an important resource for the pharmaceutical industry, but they are scarce and compromised by many technical challenges in procurement, transport, storage, and stabilization. More importantly, they are not renewable.
Development of a biomanufacturing industry for renewable normal human cells would also impact current applications based on donor harvest. Despite the universal supply of donor human blood and blood products, regional shortages occur; and the safety of the collected supply is a continual concern. In addition, certain highly desirable blood types like O-negative are always in short supply. Successful blood cell biomanufacturing based on new technologies for large-scale culture of normal human HSCs would make human blood donation obsolete and provide a blood product accessible to bioengineering of advantageous properties as well (e.g., tailored bloodtype). Similarly, the major barrier to wider availability of bone marrow cell transplantation therapy is an inadequate supply of histocompatible transplant donors. This problem is further aggravated for patients who are members of minority populations. Establishment of industries that biomanufacture panels of renewable type-specific human HSCs would advance a new era of biomedicine. The same biomanufactured HSCs could also be genetically engineered with therapeutic genes for safer gene therapies. These specific examples for blood and HSCs represent general application principles for other medically significant organs and tissues.

\section{Current Human Cell Production Technologies}

The advance of recombinant proteins as a new class of biologic medicines motivated the engineering of new technologies for manufacturing-scale fermentation of microorganisms like bacteria and yeasts and high-volume culture of mammalian cells. These technologies are highly advanced. However, many of the mammalian cells commonly employed for mass production of medical biologics are not of human origin. Instead, many are derived from other mammalian species like rodents. An even more important consideration is that all mammalian cells that can be cultured indefinitely are abnormal. Only tumor-derived or oncogenically transformed human cells can propagate sufficiently in culture to achieve the manufacturing-scale cell mass required to support a cell manufacturing industry. However, as a consequence of the genetic changes responsible for their extensive proliferative capacity, these cells have lost many of the desirable mature tissue functions for tissue cell replacement therapies or drug evaluation assays. Moreover, they are tumorigenic if transplanted into a patient. The same limitations apply to ESCs and iPSCs and mature cells derived from them (Figure 1) $[5,6]$.

Although the clinical significance of advancing cellular therapy development to mass-production of specifically normal human cells is an emerging concept, the general idea of producing human cells for therapeutic purposes is not. Though somewhat poorly informed, the latter idea has motivated the commercial development of new cell production reagents designed to allow culture of human cells that would be acceptable for clinical use. Most of this activity has been directed to developing culture media and reagents free of animalderived components, which reduces the risk of contaminating zoonotic microorganisms. These technologies provide an important starting point for beginning process engineering for large-scale production of normal human DSCs and their mature progeny cells. As is discussed in the next section, although these axenic products were developed for production of ESCs and iPSCs, the abnormalities and safety concerns of these pluripotent stem cells make their use inadvisable for drug evaluation applications and currently prohibited for cell therapy applications. 


\section{DSC Production Technologies are Best Suited for Advancing Human Cell Biomanufacturing}

Most available research reports on normal human cell production are based on small-scale laboratory experiments, of which few are focused on increasing cell yield. Even those few that have a cell yield focus lack an emphasis on bulk cell production. Typically, research studies evaluate how many times flask-size cultures can be diluted and used to serially initiate new cultures. This common cell culture procedure, called "passaging" or "splitting" provides estimates of the potential yield of cell cultures, if all cells produced were carried forward. However, in reality, there is little experience reported for producing normal human cells for clinical use in industrial-size batches on the order of $\geq 1$ trillion cells, which is equivalent to $\sim 1$ pound of cell protein. For drug evaluations, 1 trillion cells could constitute $\sim 10$ million 96well plate assays; if they were red blood cells, they would constitute $\sim 1 / 2$ unit of blood; and if they were HSCs, they might provide bone marrow transplants for more than 10,000 patients. In the U.S. alone, the average number of HSC transplants in 2012, with many unsuccessful ones due to inadequate transplant cell number, was $\sim 18,000$ [7].

Because normal mature human cells, which express the biological functions that are essential for successful drug evaluations or cell transplantation therapies, do not divide themselves, all strategies envisioned for large-scale cell production must include two main steps. The first step is the expansion of stem cells that are the precursors for mature cells. The second step is the manipulation of cell culture conditions, so that the expanded stem cells either differentiate directly into more mature cells; or they change their division pattern, so that they also produce differentiating cells [8]. Achieving complete mature cell differentiation is a critical, but often understated, challenge to optimal success in cell manufacturing. However, it is also currently one of the most intense areas of cell and tissue biology research in universities [9], which promises near term advances in knowledge for application in process engineering for the manufacture of specific normal human cell types.

For the two types of artificial stem cells, ESCs and iPSCs, selfreplication is an inherent property. ESCs are produced by the application of specialized culture media and culture conditions that promote the self-replication of specialized cells from disrupted human embryos. This requirement for the destruction of living human embryos poses an ethical barrier to the use of human ESCs (Figure 1). It has recently become evident that, part and parcel with their derivation, ESCs have genetic defects [10,11]. IPSCs, which have very similar properties to ESCs, are produced by genetic engineering of cells from mature tissues using ESC-expressed genes and oncogenes. Like ESCs, once derived, iPSCs are intrinsically actively proliferating, but they also contain worrisome genetic defects and form tumors after transplantation $[10,11]$.

The excitement about the use of ESCs and iPSCs for human cell production is due to their property of pluripotency [6]. As pluripotent stem cells, after expansion, they are able to produce differentiated cells with properties of many different types of human tissues. In the original formulation of their use for human cell production, careful removal of residual incompletely differentiated stem cells from differentiated cell populations was proposed as the processing solution to the tumor risk problem [6]. However, the past decade of pluripotent stem cell research identified two additional problems that largely cancel the envisioned advantages of pluripotency. Both new problems were predictable because of the biological processes involved. The first problem is that it is difficult to instruct ESCs and iPSCs to undergo robust differentiation into specified tissue cell types, resulting in undesirable heterogeneous cell preparations. The second, and more pervasive, problem is that the differentiated cells produced are developmentally immature. As might be expected based on the natural process of embryonic development, they are more like the cells in embryonic and fetal tissues than those found in post-natal tissues, which possess the properties essential for therapeutic efficacy [5,9]. Though these critical barriers may eventually be breached with increased scientific knowledge, presently they hold pluripotent stem cell-based human cell production in the realm of academic research, rendering it too early-stage for founding a new human cell biomanufacturing industry.

As anticipated in the previous section, DSC research has reached sufficient maturity to lead the transition from early-stage technologies to manufacturing-scale normal human cell production. The critical advance in DSC technology that makes this leadership possible is the development of methods that promote the propagation of DSCs in culture with retention of their inherent ability to produce tissue-specific mature cells $[1-4,8]$. In retrospect, the key to the paradoxical poor expansion of DSCs in culture is now self-evident. In the body, tissue DSCs only increase their number under the special circumstances of body growth and tissue repair. They primarily multiply asymmetrically, meaning they divide into sister cells that differ in their self-renewal and differentiation properties. Whether the asymmetric selfrenewal process is based on single-DSC deterministic programs or multiple-DSC stochastic programs, the net result is the same. Tissue developmental units maintain one or more DSC sisters, while the other sisters produced differentiate and undergo a limited number of divisions to produce mature tissue cells [12]. In cell culture, this type of asymmetric self-renewal by DSCs results in their dilution and loss among their more numerous differentiated progeny cells - hence, the apparent paradox. Actually DSCs divide as actively in culture as they do in the body. However, in the body, their mature progeny cells continuously complete their function, die, and are lost from the tissue. In the cell culture dish, the same maturing progeny cells accumulate, obscuring and diluting their parental DSCs $[4,8]$.

The key advance that now enables DSC biomanufacturing was the bioengineering of the SACK method to shift DSCs to a symmetric selfrenewing state reversibly. By culturing DSCs in medium supplemented with naturally occurring blood metabolites like xanthine, called SACK agents [1-4], the cells can be induced to increase self-replication without production of differentiating progeny cells. Symmetric selfrenewal promotes the rapid exponential expansion of DSCs. Once DSCs have been expanded to mass numbers, if the SACK agents are removed, the DSCs return to asymmetric self-renewal. Since the SACK agents are natural, non-mutagenic, and completely reversible, their removal promotes the induction of an even greater number of normal differentiating cells [8].

The DSC-SACK technology has been validated for rat $[1,13,14]$ and human liver DSCs [15], mouse [16] and human pancreas DSCs [4], and mouse hair follicle DSCs [3,17]. These early successes support the prediction that the universal nature of the SACK principle will make it applicable to a wide range of medically significant human tissue cells $[2,8]$. DSC-SACK overcomes the shortcomings described earlier for pluripotent stem cells (Figure 1). SACK-expanded DSCs are non-tumorigenic $[1,4,16]$, making them suitable for development for cell transplantation therapies. Both the DSCs and their induced 
differentiated progeny cells are normal, as they required no genetic manipulations for production. Because the DSCs can be induced to undergo extensive symmetric self-renewal $[1,3,15,16]$, they can be genetically engineered with therapeutic genes that are expressed in either themselves or their differentiation progeny [18]. In transplantation therapies, because DSCs are non-tumorigenic, there is no requirement for their removal from transplant preparations. In fact, for therapies like bone marrow transplantations, mixed preparations of stem cells and differentiating cells are desirable.

For drug discovery efforts, assays based on DSC-derived mature tissue cells will better reflect the complex cellular make-up of intact human tissues. Importantly, since DSCs are derived from mature post-natal tissues and are not pluripotent, they intrinsically produce mature tissue cells that are predestined to respective tissue-specific differentiation. Though not eliminating the research challenges of achieving complete mature cell differentiation altogether, this feature of the DSC-SACK technology greatly reduces this remaining barrier to ideal production of mature normal human cells for drug discovery applications (Figure 1)

\section{Establishing a Normal Human Cell Biomanufacturing Industry - Elements for Success}

Regenerative medicine is a future waiting for a particular technological advance before it can be realized. That technological advance is development of a manufacturing process for producing normal human tissue cells consistently and on-demand. With the recent advance of DSC-SACK technology, commercial supply of animal-free human cell culture components, and research on biological mechanisms of cell differentiation, much of the necessary knowledgebase for engineering innovative human cell biomanufacturing is in place. Although continued integration of research to shore up weaker areas of biological insight is certainly an important principle in moving forward, conditions are now ripe for success in this undertaking. Below, particular considerations for success are outlined.

\section{Initial Markets Choice}

Initial development efforts should identify and target biomedical applications markets with features for the most fluid integration of human cell biomanufacturing platforms and whose current commercial interests would benefit most from the routine availability of industrial-scale, high quality, normal human cells, either stem or mature. Commercial enterprises with business development plans that already depend on human cells whose supply and quality currently limit progress are ideal for early-targeted markets. These include companies in the human cells and tissue transplantation therapy space (e.g., human and porcine islet transplantation for type I diabetes), companies that harvest and distribute normal human tissue cells for the pharmaceutical industry (e.g., liver hepatocyte suppliers), and contract research organizations that conduct drug candidate evaluations for pharmaceutical companies.

\section{Start-up Incubators}

The development of human cell biomanufacturing processes must take a scaled approach. The initial steps from flasks to small-scale bulk batches (hundred billion-cell quantities) need to be well engineered and intelligently controlled in many detailed biological and physical aspects to insure that progress to manufacturing scales (quadrillion $\left[10^{15}\right]$-cells quantities) is directly enabled. Also, although general principles are expected to emerge, in many cases because of the far-ranging biological diversity of mature human tissue cells, the requirement for tissue-specific process engineering will be the norm. Therefore, the commercial start-up incubator is an ideal environment and setting for cell manufacturing process engineering and development. Especially in cluster facilities, the incubator format supports proprietary intellectual property development while still fostering productive exchanges and integration among different tissue-specific cell manufacturing platforms.

\section{Supporting Technologies}

A number of supporting technologies will figure significantly in the design of cell manufacturing processes. Among these are innovative micro- and nano-fabrication technologies and systems engineering modeling for constructing and interrogating specialized surfaces, carriers, and devices that will be instrumental in culture systems that allow coordinated expansion of stem cells in parallel with production of mature differentiated cells.

\section{User Partners}

Because end-stage regenerative medicine applications are the commercial driver of human cell biomanufacturing, it is imperative that cell manufacturing development projects engage end-user partners from the outset. The exact needs of future users must inform the cell biomanufacturing engineering process. In the ideal, the cell manufacturing development process should enhance the research and development of prospective users as well. As identified future customers for manufactured human cells, development partners will also enhance the capitalization capability of human cell manufacturing start-ups, including being potential investors.

\section{Business Models}

Many well-known service and supply business models are quite applicable for companies in the envisioned new normal human cell biomanufacturing industry. Some, like contract research organization models, are predictable. However, because of the innovation of the industry, unique models are likely to develop as well. Because of the diverse characteristics of human tissue cells, a range of company types is possible, ranging from businesses based on niche production of a single human cell type to companies that engineer the production of newly demanded cell types on a customized basis.

\section{Workforce Development}

Workforce development must walk right on the heels of the new human cell manufacturing industry. Some of the new workforce needs were already anticipated with the creation of biological engineering and chemical-biology engineering departments that began to emerge in U.S. colleges and universities about a decade or so ago. Graduates of these programs are already obtaining biotechnology and biomedicine seasoning in their early careers. Many of them have suitable training to become leaders in this new industry. However, middle-level skilled technicians for the many new technical jobs that a human cell manufacturing industry will create must be educated and trained to support future growth. As the industry is coming online, it will be important for developers to meet with educational leaders to insure visibility of the new employment opportunities and to foster science and engineering curricula developments that anticipate the preparation required for successful entry of graduates into the workforce of the new industry. 
Citation: Sherley JL (2014) Accelerating Progress in Regenerative Medicine by Advancing Distributed Stem Cell-based Normal Human Cell Biomanufacturing. Pharm Anal Acta 5: 286. doi: 10.4172/2153-2435.1000286

Page 5 of 5

\section{Conclusions}

Cell engineering now rests at a momentous opportunity to drive rapid advances in improvement of the quality of human health and life through a wide-ranging activation of the ideals of regenerative medicine. Moving from this idealistic rest to actuation requires one crucial ingredient, an industry that produces normal human cells in quantity and on-demand. Recent advances in DSC biotechnology make this development quite feasible now. In particular, the recent demonstrations of ex vivo expansion of normal human DSCs by the SACK method may open a door to routine production of other clinically important DSCs like HSCs, neural stem cells, cardiac stem cells. So, the time is now right for assembling and integrating the new DSC technologies with mature non-human mammalian cell manufacturing technology and academic and commercial interests towards realizing the full potential of regenerative medicine. This combination of knowledge and motivation can initiate a new era of biomedicine and human experience.

\section{Conflicts of Interest}

The author acknowledges that the company he leads, The Adult Stem Cell Technology Center, LLC, has a business model focused on biomanufacturing postnatal human distributed stem cells.

\section{References}

1. Lee HS, Crane GG, Merok JR, Tunstead JR, Hatch NL, et al. (2003) Clonal expansion of adult rat hepatic stem cell lines by suppression of asymmetric cell kinetics (SACK). Biotechnol Bioeng 83: 760-771.

2. Paré JF, Sherley JL (2006) Biological principles for ex vivo adult stem cell expansion. Curr Top Dev Biol 73: 141-171.

3. Huh YH, King J, Cohen J, Sherley JL (2011) SACK-expanded hair follicle stem cells display asymmetric nuclear Lgr5 expression with non-random sister chromatid segregation. Sci Rep 1: 176.

4. Paré JF, Sherley JL (2013) Ex vivo expansion of human pancreatic distributed stem cells by suppression of asymmetric cell kinetics (SACK). J Stem Cell Res Therapy 3: 149.
5. McGivern JV, Ebert AD2 (2013) Exploiting pluripotent stem cell technology for drug discovery, screening, safety, and toxicology assessments. Adv Drug Deliv Rev.

6. Lukovic D, Stojkovic M, Moreno-Manzano V, Bhattacharya SS, Erceg S (2014) Perspectives and future directions of human pluripotent stem cell-based therapies: lessons from Geron's clinical trial for spinal cord injury. Stem Cells Dev 23: 1-4.

7. U.S. Department of Health and Human Services (2013) Online Human Resources and Services Administration Blood Cell Transplant.

8. Sherley JL (2013) Advancing renewable normal human cell assays for drug discovery. Drug Devel Res 74: 127-137.

9. Cohen DE, Melton D (2011) Turning straw into gold: directing cell fate for regenerative medicine. Nat Rev Genet 12: 243-252.

10. Mummery C (2011) Induced pluripotent stem cells--a cautionary note. N Eng J Med 364: 2160-2162.

11. Jung Y, Bauer G, Nolta JA (2012) Concise review: Induced pluripotent stem cell-derived mesenchymal stem cells: progress toward safe clinical products. Stem Cells 30: 42-47.

12. Sherley JL (2013) New cancer diagnostics and therapeutics from a ninth 'hallmark of cancer': symmetric self-renewal by mutated distributed stem cells. Expert Rev Mol Diagn 13: 797-810

13. Sherley JL, Lee HS, Crane GG (2010) Hepatocyte precursor cell lines. U.S. Patent No. 7,645,610.

14. Sherley JL, Lee HS, Crane GG (2013) Hepatocyte precursor cell lines. U.S Patent No. 8,404,481.

15. Sherley JL, Panchalingam K (2010) Methods for ex vivo propagation of adult hepatic stem cells. U.S. Patent No. 7,824,912.

16. Paré JF, Sherley JL (2011) Culture environment-induced pluripotency of SACK-expanded tissue stem cells. J Biomed Biotechnol 2011: 312457.

17. Sherley JL, King J (2010) Methods for ex vivo propagation of somatic hair follicle stem cells. U.S. Patent No. 7,655,465.

18. Taghizadeh RR, Sherley JL (2008) CFP and YFP, but not GFP, provide stable fluorescent marking of rat hepatic adult stem cells. J Biomed Biotechnol 2008 453590 . 